\title{
Circulating RIPK3 levels are associated with mortality and organ failure during critical illness
}

Kevin C. Ma, ${ }^{1,2}$ Edward J. Schenck, ${ }^{1,2}$ llias I. Siempos, ${ }^{1}$ Suzanne M. Cloonan, ${ }^{1}$ Eli J. Finkelsztein, Maria A. Pabon, ${ }^{1,3}$ Clara Oromendia, ${ }^{4}$ Karla V. Ballman, ${ }^{4}$ Rebecca M. Baron, ${ }^{5}$ Laura E. Fredenburgh, ${ }^{5}$ Angelica Higuera, ${ }^{5}$ Jin Young Lee, ${ }^{6}$ Chi Ryang Chung, ${ }^{6}$ Kyeongman Jeon, ${ }^{6}$ Jeong Hoon Yang, ${ }^{6}$ Judie A. Howrylak, ${ }^{7}$ Jin-Won Huh, ${ }^{8}$ Gee Young Suh, ${ }^{6}$ and Augustine M.K. Choi ${ }^{1,2}$

'Division of Pulmonary and Critical Care Medicine, Joan and Sanford I. Weill Department of Medicine, ${ }^{2}$ NewYorkPresbyterian Hospital, ${ }^{3}$ Division of General Internal Medicine, Joan and Sanford I. Weill Department of Medicine, and ${ }^{4}$ Department of Healthcare Policy and Research, Division of Biostatistics and Epidemiology, Weill Cornell Medicine, New York, New York, USA. 'Division of Pulmonary and Critical Care Medicine, Department of Medicine, Brigham and Women's Hospital, Harvard Medical School. Boston, Massachusetts, USA. ${ }^{6}$ Department of Critical Care Medicine, Samsung Medical Center, Sungkyunkwan University School of Medicine, Seoul, Korea. 'Division of Pulmonary, Allergy, and Critical Care Medicine, Penn State Milton S. Hershey Medical Center, Hershey, Pennsylvania, USA. ${ }^{8}$ Department of Pulmonary and Critical Care Medicine, Asan Medical Center, University of Ulsan College of Medicine, Seoul, Korea.

BACKGROUND. Necroptosis is a form of programmed necrotic cell death that is rapidly emerging as an important pathophysiological pathway in numerous disease states. Necroptosis is dependent on receptor-interacting protein kinase 3 (RIPK3), a protein shown to play an important role in experimental models of critical illness. However, there is limited clinical evidence regarding the role of extracellular RIPK3 in human critical illness.

METHODS. Plasma RIPK3 levels were measured in 953 patients prospectively enrolled in 5 ongoing intensive care unit (ICU) cohorts in both the USA and Korea. RIPK3 concentrations among groups were compared using prospectively collected phenotypic and outcomes data.

RESULTS. In all 5 cohorts, extracellular RIPK3 levels in the plasma were higher in patients who died in the hospital compared with those who survived to discharge. In a combined analysis, increasing RIPK3 levels were associated with elevated odds of in-hospital mortality (odds ratio [OR] 1.7 for each $\log _{10}$-unit increase in RIPK3 level, $P<\mathbf{0 . 0 0 0 1}$ ). When adjusted for baseline severity of illness, the OR for in-hospital mortality remained statistically significant (OR 1.33, $P=0.007$ ). Higher RIPK3 levels were also associated with more severe organ failure.

CONCLUSIONS. Our findings suggest that elevated levels of RIPK3 in the plasma of patients admitted to the ICU are associated with in-hospital mortality and organ failure.

FUNDING. Supported by NIH grants P01 HL108801, R01 HL079904, R01 HL055330, R01 HL060234, K99 HL125899, and KL2TR000458-10. Supported by Samsung Medical Center grant SMX1161431.

Conflict of interest: The authors have declared that no conflict of interest exists.

Submitted: January 8, 2018

Accepted: June 6, 2018

Published: July 12, 2018

Reference information: JCI Insight. 2018;3(13):e99692. https://doi.org/10.1172/jici. insight.99692.

\section{Introduction}

The recent Sepsis-3 guidelines changed the conceptual framework and definition of sepsis and highlighted the importance of organ dysfunction as a hallmark of the syndrome (1). Organ dysfunction during critical illness often reflects an abnormal systemic inflammatory response to external triggers and is independently associated with poor outcomes. Specifically, infected patients with organ dysfunction have a 2- to 25-fold increased risk of dying in the hospital compared with infected patients without organ dysfunction (2). Among mixed critically ill patients, the degree of organ dysfunction is highly correlated with mortality (3). Despite these observations, the identification of patients at risk for such organ dysfunction and the precise pathobiology associated with such organ injury remain unknown. 


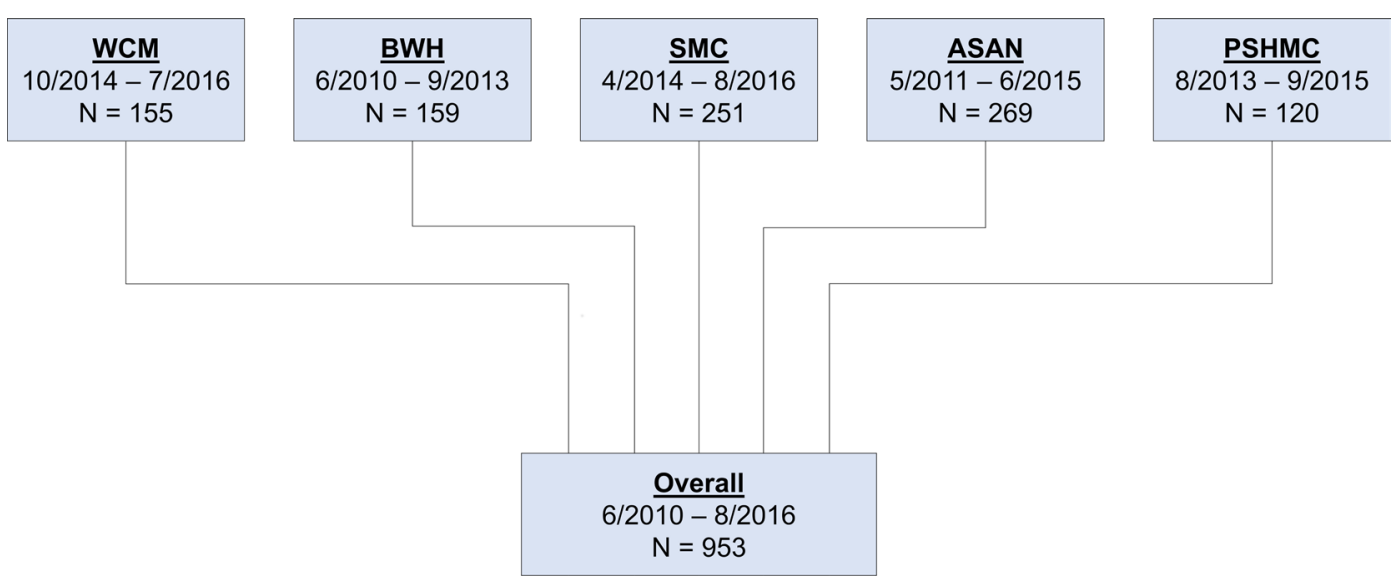

Figure 1. Number of recruited subjects and date ranges among individual cohorts and overall. WCM, Weill Cornell Medicine; BWH, Brigham and Women's Hospital; SMC, Samsung Medical Center; ASAN, Asan Medical Center; PSHMC, Penn State Hershey Medical Center.

Cell death plays a role in the pathogenesis of organ dysfunction during critical illness (2). Nonspecific cellular injury leads to necrosis. Necrotic cell death not only creates local organ injury, but releases proinflammatory endogenous danger signals termed damage-associated molecular patterns (DAMPs). DAMPs are often molecules including proteins that have separate intracellular roles in cell homeostasis during noninflammatory states. However, the release of these molecular patterns in the extracellular space after necrotic cell death stimulates the innate immune response through binding of pathogen recognition receptors $(4,5)$. Excessive release of DAMPs in the setting of critical illness may create an ongoing inflammatory cascade leading to further cell death and organ injury (6-8). Elevated DAMPs have been associated with increased mortality in multiple critical illnesses including sepsis, trauma, cardiac arrest, and other pathophysiological states (9). Apoptosis, the traditionally understood form of programmed cell death, is characterized by an ordered dismantling of the cell into small vesicles or apoptotic bodies. Apoptosis has been observed during critical illness but is immunologically silent $(10,11)$.

Recently, our understanding of programed cell death has expanded to include forms of regulated, or programed, necrosis. Necroptosis, one subtype of programed necrosis, occurs after stimulation with proinflammatory cytokines such as TNF- $\alpha$ when apoptotic pathways are blocked (12). Necroptosis occurs through the formation of the necrosome, a 3-protein complex including receptor-interacting protein kinase 1 (RIPK1), RIPK3, and mixed-lineage kinase domain-like protein (MLKL) (13). The necroptosis-related enzyme RIPK3 has been shown to play an important role in experimental models of critical illnesses, including, but not limited to, acute lung injury, pneumococcal pneumonia, and acute kidney injury (14-16). However, there is limited clinical evidence regarding the role of necroptosis in human critical illness (15-17). We therefore sought to explore the association between RIPK3 levels in the plasma at the time of admission to the ICU and mortality during the index hospitalization across multiple independent cohorts in the largest study to date of this novel biomarker in critical illness. We also sought to examine the correlation between RIPK3 levels in the plasma with the severity of organ failure. Our goal was to provide evidence that increased circulating RIPK3 is associated with higher in-hospital mortality and morbidity, suggesting necroptosis may be an important cell death pathway during critical illness.

Some of the results of this study have been previously reported in the form of abstracts $(18,19)$.

\section{Results}

A total of 953 subjects across 5 ICU cohorts, from 2 continents were included in the study (Figure 1). Baseline characteristics of all 953 subjects are presented in Table 1. Four of the cohorts (NewYork-Presbyterian Hospital/Weill Cornell Medicine [WCM], Brigham and Women's Hospital [BWH], Asan Medical Center [ASAN], and Penn State Milton S. Hershey Medical Center [PSHMC]) were medical ICUs, while one cohort (Samsung Medical Center [SMC]) was a mixed medical and cardiac ICU. Among all 5 cohorts, 
Table 1. Baseline demographics and clinical characteristics among individual cohorts and overall

\begin{tabular}{|c|c|c|c|c|c|c|}
\hline Characteristic & WCM & BWH & SMC & ASAN & PSHMC & Overall \\
\hline Male, $n(\%)$ & $83(54)$ & $92(58)$ & $171(68)$ & $178(66)$ & $68(57)$ & $592(62)$ \\
\hline \multicolumn{7}{|l|}{ Race } \\
\hline White, $n(\%)$ & $96(62)$ & $118(74)$ & $0(0)$ & $0(0)$ & $110(92)$ & 324 (34) \\
\hline Asian, $n(\%)$ & $6(4)$ & $3(2)$ & $251(100)$ & $269(100)$ & $0(0)$ & $528(55)$ \\
\hline Other, $n(\%)$ & $13(8)$ & $4(3)$ & $0(0)$ & $0(0)$ & $3(3)$ & $20(2)$ \\
\hline \multicolumn{7}{|l|}{ Diagnoses } \\
\hline $\begin{array}{l}\text { Infected without organ } \\
\text { failure, } n(\%)\end{array}$ & $4(3)$ & 14 (9) & $0(0)$ & $1(0.4)$ & $1(1)$ & $20(2)$ \\
\hline Sepsis, $n(\%)$ & $79(51)$ & $77(48)$ & $79(32)$ & $145(54)$ & $68(57)$ & $447(47)$ \\
\hline Noninfected with shock, $n$ (\%) & $6(4)$ & $3(2)$ & $17(7)$ & $0(0)$ & $6(5)$ & $32(3)$ \\
\hline \multicolumn{7}{|l|}{ Interventions } \\
\hline Use of vasopressors, $n$ (\%) & $72(47)$ & $51(32)$ & $124(49)$ & $193(72)$ & $47(39)$ & $486(51)$ \\
\hline $\begin{array}{l}\text { Use of mechanical ventilation, } \\
n(\%)\end{array}$ & $51(33)$ & $48(30)$ & 98 (39) & $164(61)$ & $77(76)$ & $439(47)$ \\
\hline Lactate mg/dl, median (IQR) & $1.9(1.2-3.8)$ & $2.1(1.4-2.9)$ & $2.2(1.4-3.7)$ & $2.1(1.3-4.4)$ & $1.7(1.1-2.5)$ & $2.0(1.3-3.6)$ \\
\hline APACHE II score, median (IQR) & $22(17-28)$ & $25(19-30)$ & $20(13-26)$ & $24.0(19-28)$ & $24(18-31)$ & $23(17-28)$ \\
\hline SOFA score, median (IQR) & $7(5-10)$ & $6(3-8)$ & $6(3-10)$ & $9(7-12)$ & $7(5-11)$ & $7(5-11)$ \\
\hline In-hospital mortality, n (\%) & $31(20)$ & $23(15)$ & $44(18)$ & 87 (33) & $37(31)$ & $222(23)$ \\
\hline
\end{tabular}

IQR, interquartile range; APACHE II, acute physiology and chronic health evaluation II; SOFA, sequential organ failure assessment; WCM, Weill Cornell Medicine; BWH, Brigham and Women's Hospital; SMC, Samsung Medical Center; ASAN, Asan Medical Center; PSHMC, Penn State Hershey Medical Center.

$592(62 \%)$ subjects were male with a median age of 65 years (interquartile range [IQR] 53-74). All subjects in the 2 Korean cohorts were of Asian descent, while almost all patients in the PSHMC cohort were of European descent (92\%). In the other 2 American cohorts, $62 \%(\mathrm{WCM})$ and $74 \%(\mathrm{BWH})$ of subjects were of European descent.

Infection was the most common reason for ICU admission in all cohorts, although frequency differed to a certain degree (overall: $76 \%[n=726]$, WCM: $78 \%$ [ $n=121]$, BWH: $74 \%[n=117]$, SMC: $57 \%[n=$ 142], ASAN: 98\% [ $n=264]$, PSHMC: $68 \%$ [ $n=82]$ ). Certain cohorts had unique differences in baseline demographics. Compared with the combined group, the PSHMC cohort had disproportionally more subjects with acute respiratory distress syndrome (33\% vs. $18 \%)$ and more patients on mechanical ventilation (76\% vs. 47\%). The ASAN cohort was composed predominantly of subjects with sepsis (98\%), with $44 \%$ having septic shock $(n=44)$. The in-hospital mortality of the 5 cohorts combined was $23 \%(95 \%$ confidence interval $[\mathrm{CI}] 21 \%-26 \% ; n=223)$. The BWH cohort had the lowest $(15 \%, n=23)$, while the ASAN cohort had the highest $(33 \%, n=88)$ in-hospital mortality.

Median acute physiology and chronic health evaluation (APACHE) II score at time of ICU admission was between 20 (SMC) and 25 (BWH), overall 23 (IQR 17-28). Median sequential organ failure assessment (SOFA) score was between 6 and 9, overall 7 (Table 1). The median composite SOFA score in each cohort is shown in Table 1 (overall: 7 [IQR 5-11], WCM: 7 [IQR 5-10], BWH: 6 [IQR 3-8], SMC: 6 [IQR 3-10], ASAN: 9 [IQR 7-12], PSHMC: 7 [IQR 5-11]). The distribution SOFA scores among all 5 cohorts is shown in Figure 2.

Mortality. There was variation in the median and distribution of RIPK3 concentration in 2 cohorts (PSHMC: 1,886 pg/ml, BWH: 2,124 pg/ml) compared with the other 3 (WCMC: $678 \mathrm{pg} / \mathrm{ml}, \mathrm{SMC}: 380 \mathrm{pg}$ / $\mathrm{ml}$, ASAN: $512 \mathrm{pg} / \mathrm{ml}$ ) (Figure 3). In each of the cohorts, RIPK3 concentration in the plasma was higher in patients who died in the hospital compared with those who survived to discharge (median overall: 1,296 pg/ml 


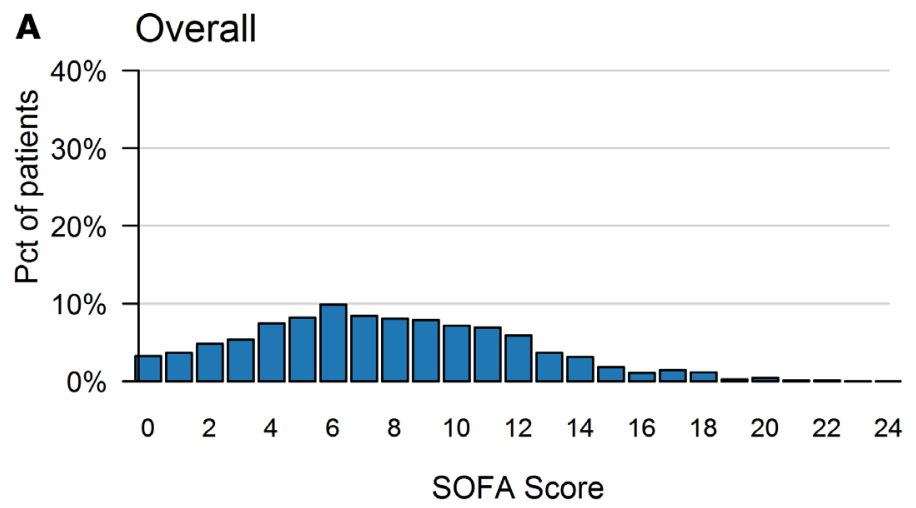

\section{B WCM}

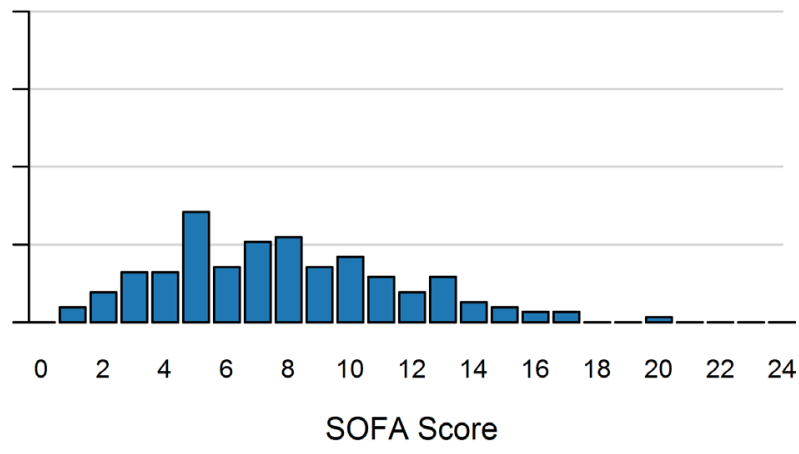

D $\mathrm{SMC}$
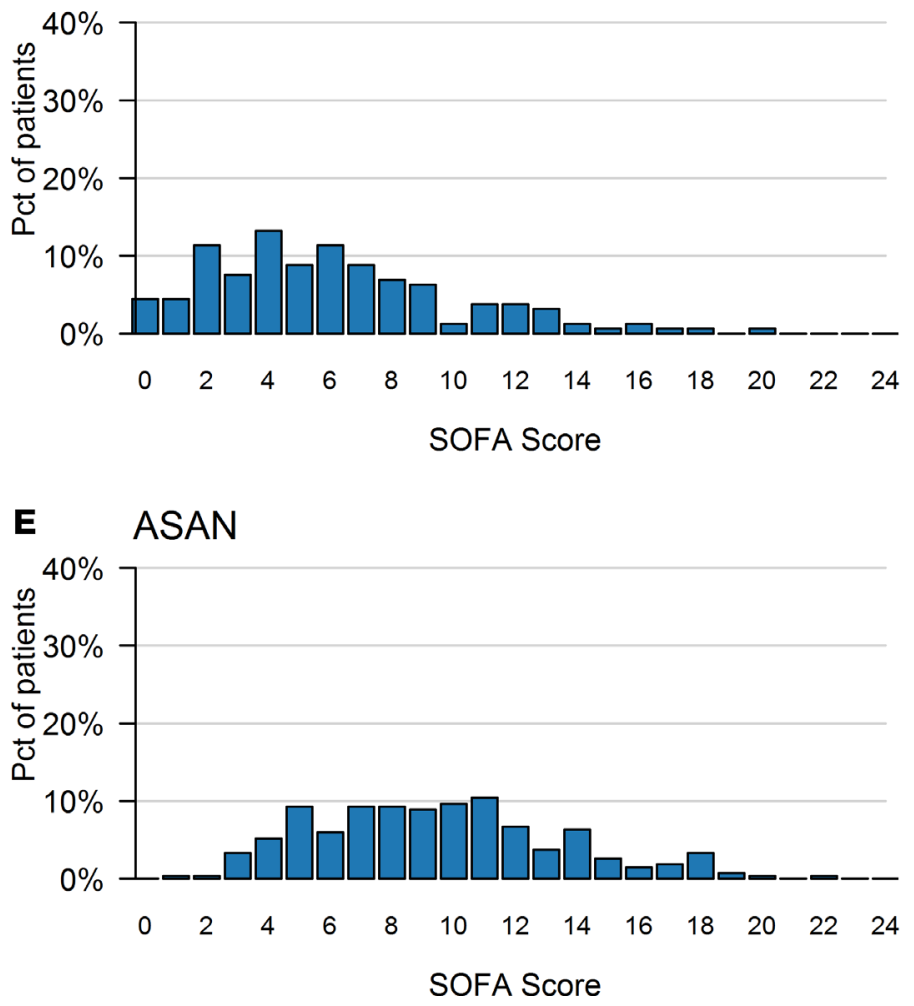

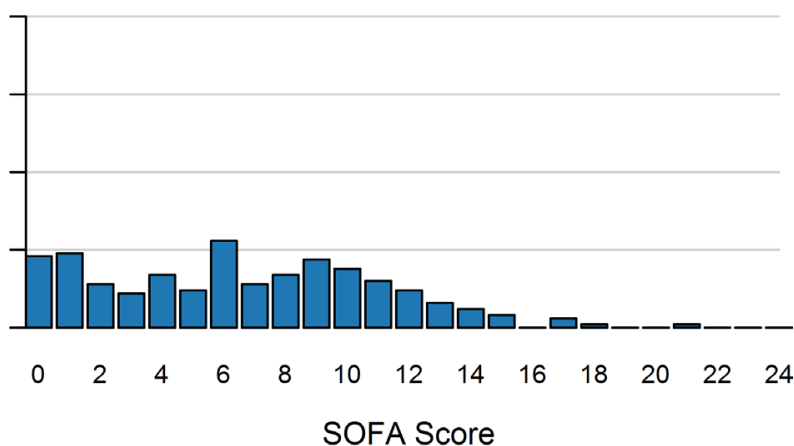

F PSHMC

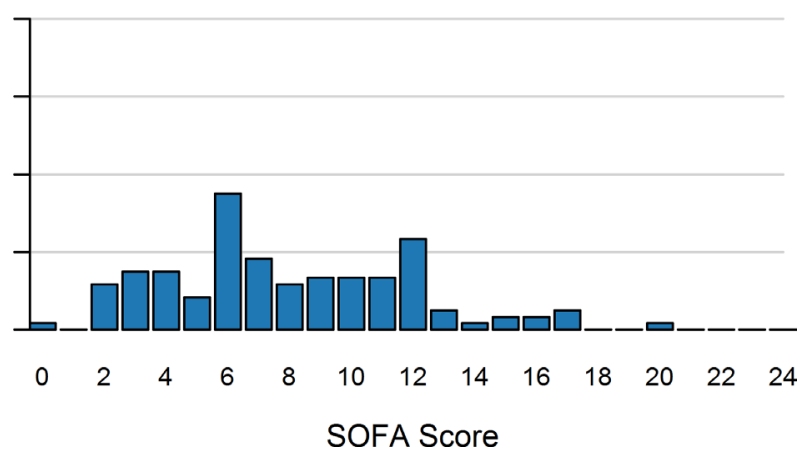

Figure 2. Distribution of SOFA score among individual cohorts and overall. SOFA, sequential organ failure assessment; WCM, Weill Cornell Medicine; BWH, Brigham and Women's Hospital; SMC, Samsung Medical Center; ASAN, Asan Medical Center; PSHMC, Penn State Hershey Medical Center.

vs. $685 \mathrm{pg} / \mathrm{ml}[P<0.001]$, WCM: $1,164 \mathrm{pg} / \mathrm{ml}$ vs. $600 \mathrm{pg} / \mathrm{ml}[P=0.016]$, BWH: $2,801 \mathrm{pg} / \mathrm{ml}$ vs. $2,113 \mathrm{pg} /$ $\mathrm{ml}[P=0.091]$, SMC: $899 \mathrm{pg} / \mathrm{ml}$ vs. $296 \mathrm{pg} / \mathrm{ml}[P<0.001]$, ASAN: $858 \mathrm{pg} / \mathrm{ml}$ vs. $340 \mathrm{pg} / \mathrm{ml}[P=0.002]$, PSHMC: $4,953 \mathrm{pg} / \mathrm{ml}$ vs. $1,340 \mathrm{pg} / \mathrm{ml}[P<0.001]$ ) (Figure 3). When all 5 cohorts were examined together, subjects had a 73\% increase in the odds of dying in the hospital for each 10-fold increase in RIPK3 level, (odds ratio [OR] $1.7[1.4 \%-2.195 \% \mathrm{CI}], P<0.0001$ ) (Figure 3). When adjusted for APACHE II score, the OR for in-hospital death remained statistically significant at $1.3[1.0-1.6](P=0.007)$ (Table 2). The unadjusted OR for in-hospital death for each individual cohort ranged from 1.5 to $4.6(P<0.05$ for all, see Supplemental Table E1; supplemental material available online with this article; https://doi.org/10.1172/jci.insight.99692DS1). After adjusting for APACHE II score, the OR for death in the hospital remained statistically significant in the 2 cohorts with the largest number of events, the SMC (OR $3.1[1.0-1.895 \% \mathrm{CI}], P=0.002)$ and ASAN (OR $1.4[1.0-1.895 \% \mathrm{CI}], P=0.027$ ) cohorts (see Supplemental Table E2). All 3 remaining cohorts had numerically increased odds for in-hospital mortality among those with higher RIPK3 concentration, though statistically significance was not achieved. 


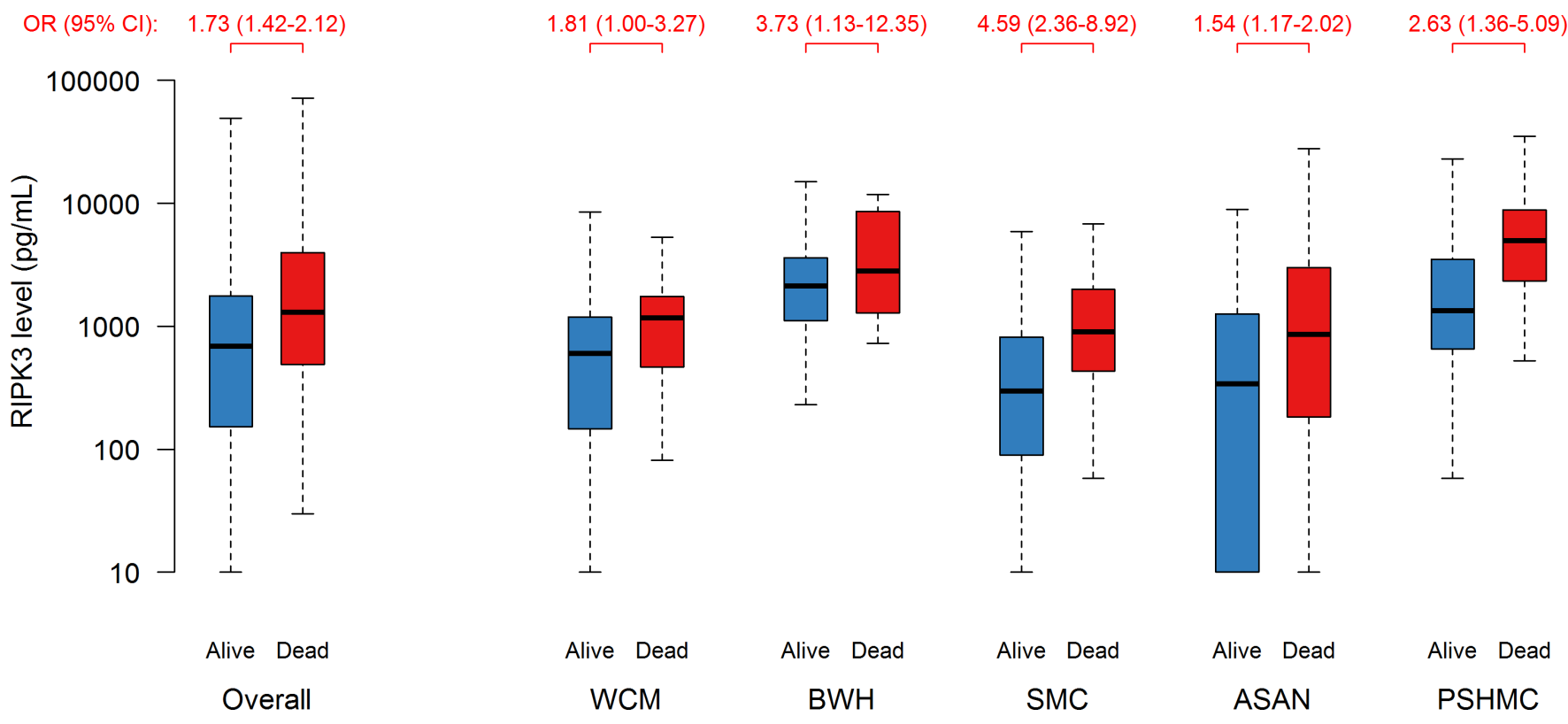

Figure 3. Association between RIPK3 level and in-hospital mortality. Odds ratios and 95\% confidence intervals represent increase in risk of in-hospital mortality associated with a 10-fold rise in RIPK3 level. RIPK3 level is presented as median value (black line), interquartile range (box), and maximum and minimum values (whiskers) overall and among individual cohorts. WCM, Weill Cornell Medicine; BWH, Brigham and Women's Hospital; SMC, Samsung Medical Center; ASAN, Asan Medical Center; PSHMC, Penn State Hershey Medical Center.

Organ failure. The relationship between RIPK3 and SOFA was best approximated with a quadratic fit for log-RIPK3. There was a statistically significant positive association between RIPK3 concentration and SOFA score on day 1 of admission to the ICU $\left(R^{2}=0.17\right)$ (Figure 4$)$. When examined based on individual components of the SOFA score, this association was also observed in all 6 components (Figure 5). In detail, RIPK3 levels were associated with all components of the SOFA score (F-statistic $P<0.001)$, with the strongest association found in renal and neurologic components $\left(R^{2}=0.096\right.$ and 0.084 , respectively) (Figure 5). While the respiratory component increased with increased RIPK3 levels, it had the weakest association, with an $R^{2}$ of 0.023 (Figure 5). When all 5 cohorts were examined together, RIPK3 levels in the plasma were higher in patients with organ failure and shock compared with those with organ failure but without shock or those without organ failure $(P<0.0001$ for all) (Figure 6). We noted that a 10-fold increase in RIPK3 level was associated with a $34 \%$ increase $(26 \%-42 \% 95 \% \mathrm{CI})$ in lactate (Supplemental Figure E1). No association was found between RIPK3 level and plasma lactate dehydrogenase (LDH) level (Supplemental Figure E2).

\section{Discussion}

By using data from 5 different independent ICU cohorts composed of more than 950 subjects across 2 continents, we found that elevated plasma levels of RIPK3 were associated with in-hospital mortality of critically ill patients. We also found that elevated RIPK3 levels were associated with severity of up-front organ failure as measured by the composite SOFA score as well as each of its 6 components.

Table 2. Univariate and multivariate logistic regression of RIPIK3 level and mortality for all 5 cohorts combined

\begin{tabular}{lcccc}
\hline & \multicolumn{2}{c}{ Univariable } & \multicolumn{2}{c}{ Multivariable } \\
& OR $(95 \% \mathrm{CI})$ & $P$ value & OR $(95 \% \mathrm{Cl})$ & $P$ value \\
APACHE II & - & - & $1.08(1.05-1.10)$ & $<0.0001$ \\
$\log _{10}$ RIPK3 & $1.73(1.42-2.12)$ & $<0.0001$ & $1.33(1.08-1.64)$ & 0.0066
\end{tabular}

APACHE II, acute physiology and chronic health evaluation II; OR, odds ratio; Cl, confidence interval. 


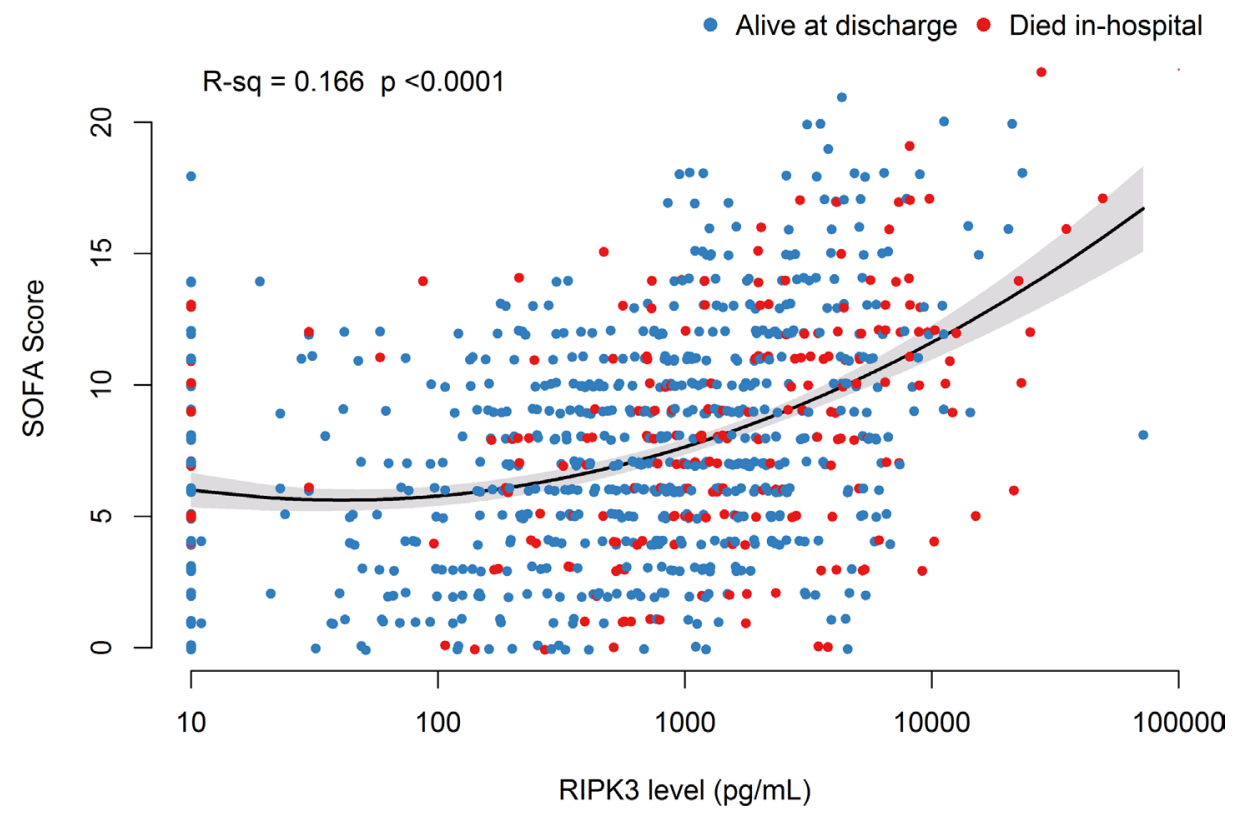

Figure 4. Association between RIPK3 level and organ failure measured by SOFA score. Regression curve for quadratic modeling of RIPK3 association with SOFA score shown in black line, with shaded area representing $95 \%$ point-wise confidence interval.

Our main result regarding the association between RIPK3 levels and mortality in critically ill patients corroborate the preliminary findings of 2 single-center smaller studies $(15,17)$. In detail, Qing et al. sampled the plasma of 37 patients with sepsis and noted that nonsurvivors had higher RIPK3 levels on the second day of their ICU admission compared with survivors (15). The same research group subsequently sampled the plasma of 80 predominantly male patients with trauma and noted that RIPK 3 concentrations on the second day of their ICU admission were associated with mortality (17). Taken together, the findings of the above-mentioned contributions and our larger study, which involved individuals with a diverse range of ethnicities and clinical diagnoses, provide compelling evidence that plasma RIPK3 levels are indeed associated with mortality in the context of critical illness.

Our finding regarding the association between RIPK3 levels and organ failure seems to be robust, as it persists across each of the 5 cohorts and for all components of SOFA. Given that RIPK3 is a key molecule involved in necroptotic cell death, the revealed association between RIPK3 and organ failure raises thoughts regarding the association between organ failure and cell death. Patients with sepsis or trauma and MODS are noted to have increased markers of apoptosis in distal parenchymal tissue as well as lymphocytes (10, 20). The paradigm has been challenged in recent years with new findings suggesting that necrosis may occur in response to specific signals in the cellular milieu (21). Necroptosis is the prototype pathway of this new class of cell death but emerging mechanisms including ferroptosis, pyroptosis, parthanatos, and others are rapidly expanding our understanding of cell death (21). Regulated necrosis pathways such as necroptosis may contribute to the creation of feedback loops leading to ongoing organ failure after initial injury (22).

It should be emphasized that elevated plasma levels of RIPK3 may not necessarily represent necroptosis (23). Beyond its role as a key protein of necroptosis, RIPK3 has other important roles (23). For example, it has been shown to activate caspase-8-dependent apoptosis independent of its kinase function $(13,24,25)$. Also, RIPK3 can activate the NLRP3 inflammasome and subsequent release of inflammatory cytokine IL-1 $\beta$ in the absence of necroptotic activity (26). Moreover, the lack of a gold-standard assay for the detection of necroptotic activity adds to the uncertainty as to whether the elevated RIPK3 levels found in critically ill patients indicate necroptosis. We have recently shown that RIPK3 is elevated in subjects receiving mechanical ventilation in the ICU. In murine models, RIPK3 deficiency, but not MLKL deficiency, ameliorated physiologic, histologic, and biochemical markers of ventilator-induced lung injury (VILI). In both humans and mice, VILI was associated with impaired fatty acid oxidation, but in mice this association was not observed under conditions of RIPK3 deficiency. These findings suggest that fatty acid oxidation-dependent RIPK3 mediates the pathogenesis of 


\section{A Respiratory}

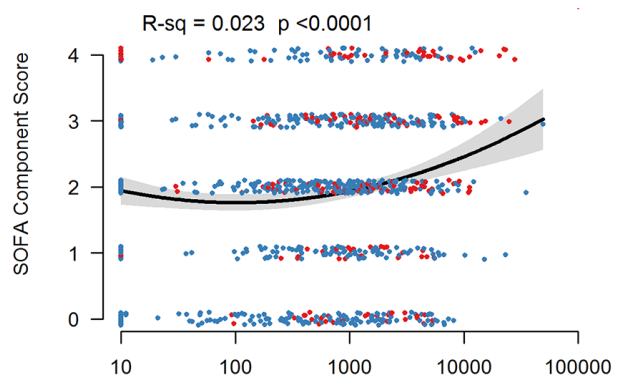

B Cardiovascular

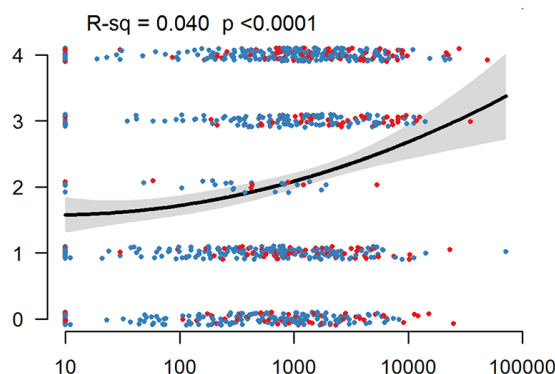

\section{Neurologic}

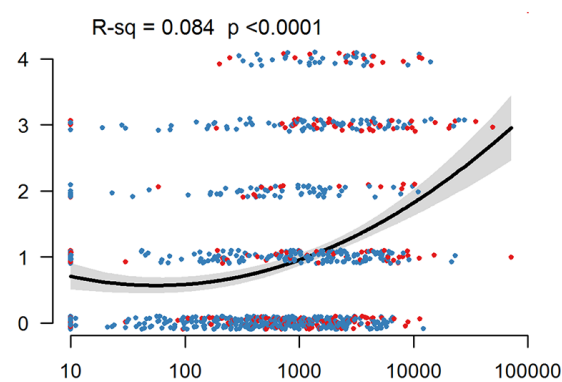

D Hematologic

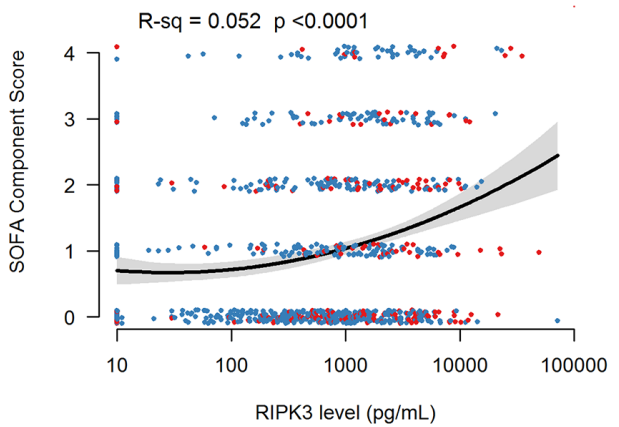

E Renal

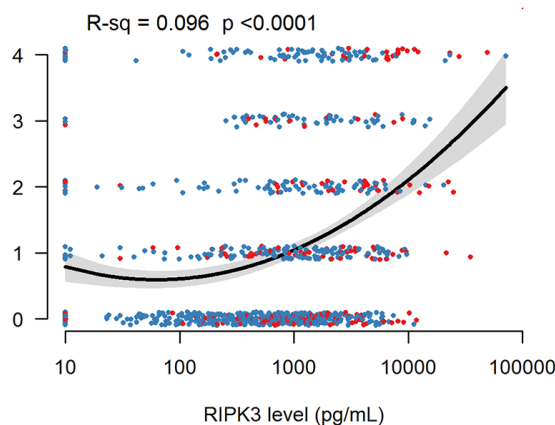

\section{F Hepatic}

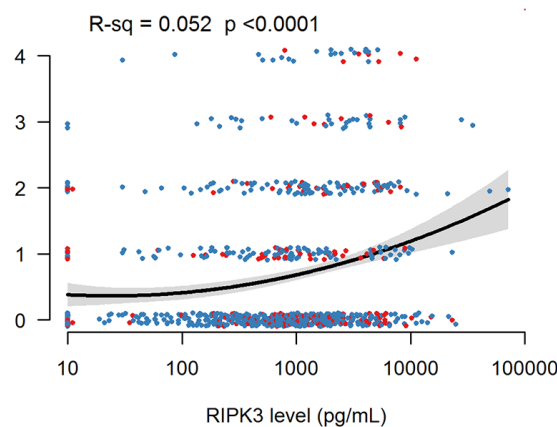

- Alive at discharge - Died in-hospital

Figure 5. Association between RIPK3 level and individual SOFA components. The components evaluated were (A) respiratory, (B) cardiovascular, (C) neurologic, (D) hematologic, (E) renal, and (F) hepatic organ systems. Regression curve for quadratic modeling of RIPK3 association with each SOFA component is shown by the black line, with shaded area representing 95\% point-wise confidence interval.

acute lung injury that may be independent of its role in necroptotic cell death (16). Additional studies are required to further elucidate whether the cell death-related or other properties of RIPK3 (e.g., inflammationrelated) are relevant in the context of human critical illness.

Our study has certain weaknesses. Firstly, we noted variation in the median and distribution of RIPK3 concentration across our cohorts (Figure 3). This variation could not be explained by admission diagnosis or disease severity. Given the geographical distribution in our cohorts, we examined whether ethnicity could be a cause but found no association between ethnicity and RIPK3 level. Secondly, there was also heterogeneity in minimum hemoglobin concentration criteria in subject recruitment. The WCM cohort utilized a hemoglobin concentration of $7 \mathrm{gm} / \mathrm{dl}$, while the BWH and SMC cohorts used a hemoglobin concentration of $8 \mathrm{gm} / \mathrm{dl}$. The ASAN and PSHMC cohorts utilized no specific hemoglobin concentration cutoff. These variations are due to our evolving understanding of the role of anemia in critical illness and changing practice patterns in blood transfusion. In a subgroup analysis of subjects with a hemoglobin value less than $8 \mathrm{gm} / \mathrm{dl}$, we found no relationship between RIPK3 and hemoglobin. Thus, we believe this difference in inclusion criteria does not contribute to any variation in the RIPK3 levels between cohorts. We hypothesize that differences in blood collection timing and plasma preparation protocol across sites may have affected the amount of RIPK3 measured in the plasma samples. Furthermore, the differences may also be due to variation in age of the plasma samples between cohorts. Thirdly, we could not know the source or mechanism of increased RIPK 3 in the plasma of critically ill patients, i.e., whether it is passive release from dying cells and/or an active secretion in response to a stimulus. Importantly, it is unclear whether the elevation in plasma RIPK3 concentration is only from release of existing RIPK3 from the cytosolic space to the plasma or whether there is upregulation of the RIPK3 expression. In a recent study by Davenport et al. examining the transcriptome of leukocytes of patients admitted to the ICU with community-acquired pneumonia, a subgroup of patients with high gene expression of RIPK3 (and other genes) was associated with higher mortality (27). These data suggest that our observations of elevated plasma RIPK3 may be in part due 


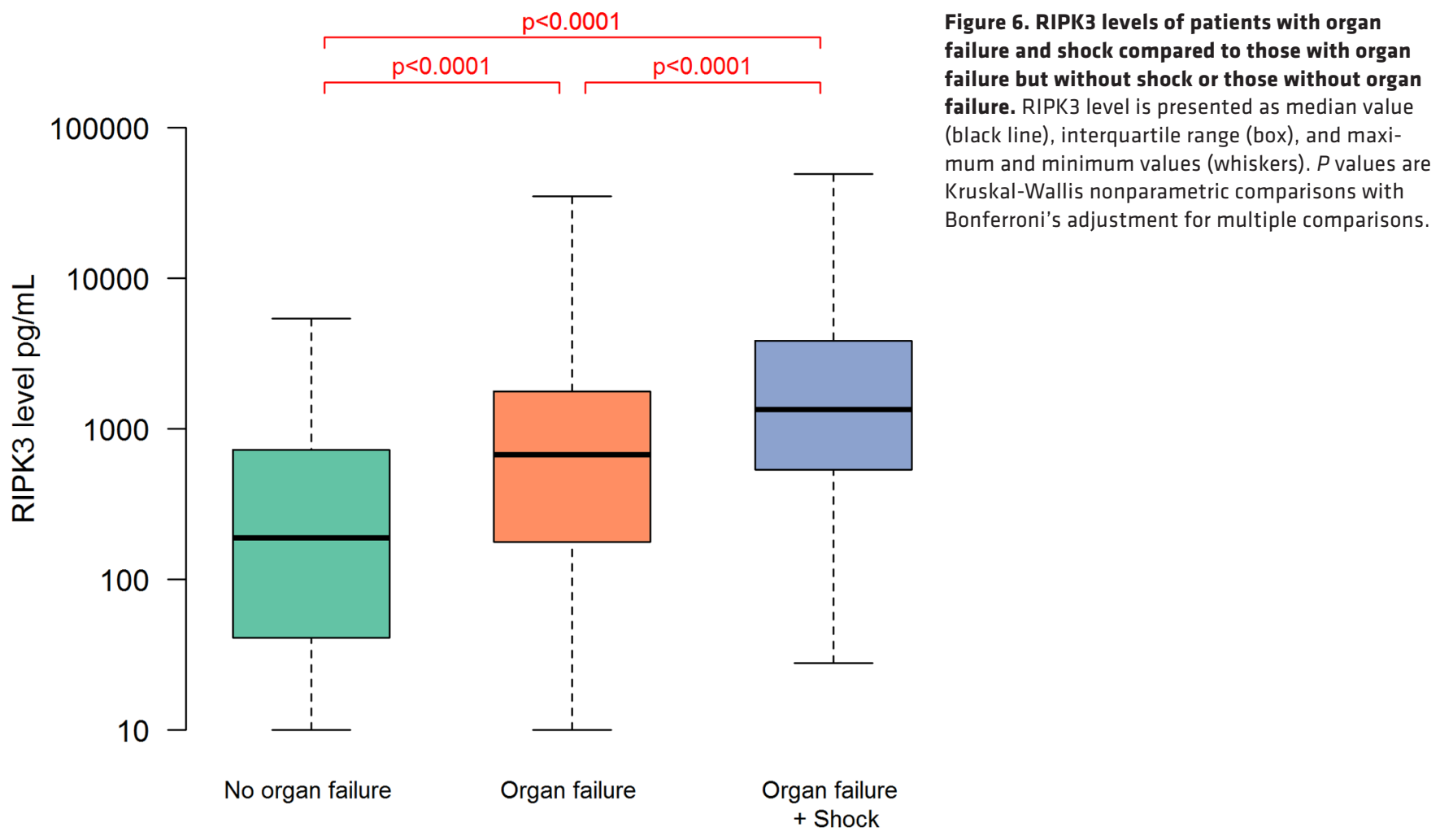

to higher expression of RIPK3 within peripheral leukocytes, rather than simple release of existing cytosolic RIPK3 (27). This is supported by the fact that we found no correlation between plasma RIPK3 level and plasma LDH level (Supplemental Figure E2).

In conclusion, our findings suggest that elevated levels of RIPK3 in the plasma of patients admitted to the ICU are associated with in-hospital mortality and organ failure.

\section{Methods}

Subjects were drawn from 5 ongoing prospective cohorts of critically ill patients admitted in ICU of the following hospitals: NewYork-Presbyterian Hospital/Weill Cornell Medicine (WCM), Brigham and Women's Hospital (BWH), Samsung Medical Center (SMC), Asan Medical Center (ASAN), and Penn State Milton S. Hershey Medical Center (PSHMC). The protocols of patient recruitment and data collection for each of 5 cohorts have been described previously (28-31) and are discussed in detail in the Supplemental text. All 5 cohorts were designed and began recruitment prior to the development of this specific study. Variations in patient inclusion and exclusion to each prospective cohort are due to local differences in the local IRB requirements and evolving practice patterns at time of development.

In brief, patients were prospectively recruited into each individual cohort upon admission to the ICU by their respective research teams. Baseline demographics, clinical details, and outcomes were recorded. Assessments of disease severity were measured using the APACHE II and SOFA scores based on values collected over the first 24 hours of admission to the ICU $(32,33)$. The recent Sepsis-3 definitions were used to distinguish patients with sepsis, septic shock, and those with infection who did not meet the above criteria (1). Similarly, patients admitted to the ICU without infection were further classified as without organ failure (SOFA score $<2$ ), having organ failure (SOFA score $\geq 2$ ), or organ failure with shock (SOFA score $\geq 2$, lactate $\geq 2 \mathrm{mg} / \mathrm{dl}$, and need for vasopressors to maintain a mean arterial pressure of $65 \mathrm{mmHg}$ or greater in the absence of hypovolemia).

All plasma samples and clinical information obtained for the study were contributed by the study investigators. Samples and clinical information from WCM were obtained from A.M.K. Choi. Samples and clinical information from BWH were obtained from R.M. Baron. Samples and clinical information from SMC were obtained from G.Y. Suh. Samples and clinical information from PSHMC were obtained from J.A. Howrylak. Samples and clinical information from ASAN were obtained from J.W. Huh. All reagents, assays, and other materials were obtained by study investigators through commercial vendors as described. 
Outcomes. All-cause mortality assessed at hospital discharge (i.e., in-hospital mortality) was the primary outcome of the study. Organ failure served as secondary outcome of the study. Organ failure was quantified by using the SOFA score. The SOFA is a 6-component scoring system ranging from 0 (best) to 24 (worst) that describes the organ function of the central nervous, cardiovascular, respiratory, renal, hepatic, and hematologic systems. Individual organ failure elements are scored from 0 (best) to 4 (worst) and combined for the composite score (33). Missing elements were noted and scored as 0 .

Measurement of RIPK3. The process of plasma isolation protocols for each individual cohort is discussed in detail in the Supplemental text. Blood plasma samples were obtained following recruitment. RIPK3 concentration was measured using a commercially available enzyme-linked immunosorbent assay (ELISA) kit as per the manufacturer's instructions (CSB-EL019737HU, CUSABIO).

Statistics. RIPK3 concentration was analyzed after a log transformation. Survival was assessed as a binary variable based on status at time of discharge from the hospital, and logistic regression used to estimate the association between outcomes and RIPK3, with or without APACHE II. Association with organ failure as measured by SOFA score was studied using linear regression. Due to the nonlinear association between logRIPK3 and SOFA scores, a model also including a quadratic term for log-RIPK3 was used. Similar analyses were performed for each SOFA component (platelets, bilirubin, creatinine, respiratory, Glasgow comma score, and cardiovascular). All analyses were conducted in R (R Foundation for Statistical Computing), and $P<0.05$ was considered statistically significant, and 95\% CIs presented where appropriate.

Study approval. The study protocol was approved by Institutional Review Boards of WCM (1405015116A005), BWH (2008-P-000495), SMC (2012-12-033), ASAN (2011-0001), and PSHMC (PRAMS041724EP). All patients or their surrogate provided written informed consent.

\section{Author contributions}

KCM, AMKC, EJS, IIS, and SMC contributed to the conception and design of the study. KCM, EJS, EJF, MAP, RMB, LEF, AH, JYL, CRC, KJ, JHY, JAH, JWH, and GYS contributed to the collection of clinical data and biological samples. KCM, EJF, MAP, SMC, and JYL contributed to the performing of assays. $\mathrm{KCM}$, EJS, CO, and KVB performed all statistical analysis. KCM contributed to the drafting of the manuscript. KCM, EJS, IIS, CO, SMC, and AMKC contributed to the editing of manuscript. CO contributed to the creation of the figures. KCM, EJS, and IIS contributed to the editing of the figures. All authors contributed to the final approval of all submitted contents.

\section{Acknowledgments}

The authors would like to acknowledge valuable discussion and input from Kiichi Nakahira (Weill Cornell Medicine) and Jong Seok Moon (Weill Cornell Medicine).

Address correspondence to: Augustine M.K. Choi, 1300 York Avenue, Suite F-113 Box 83, New York, New York 10065, USA. Phone: 212.746.6005; Email: amc2056@med.cornell.edu.

KCM's present address is: Division of Pulmonary, Allergy, and Critical Care Medicine, University of Pennsylvania, Philadelphia, Pennsylvania, USA.

1. Singer M, et al. The third international consensus definitions for sepsis and septic shock (Sepsis-3). JAMA. 2016;315(8):801-810

2. Seymour CW, et al. Assessment of clinical criteria for sepsis: For the third international consensus definitions for sepsis and septic shock (Sepsis-3). JAMA. 2016;315(8):762-774.

3. Ferreira FL, Bota DP, Bross A, Mélot C, Vincent JL. Serial evaluation of the SOFA score to predict outcome in critically ill patients. JAMA. 2001;286(14):1754-1758.

4. Matzinger P. Tolerance, danger, and the extended family. Annu Rev Immunol. 1994;12:991-1045.

5. Yatim N, Cullen S, Albert ML. Dying cells actively regulate adaptive immune responses. Nat Rev Immunol. 2017;17(4):262-275.

6. Lotze MT, Tracey KJ. High-mobility group box 1 protein (HMGB1): nuclear weapon in the immune arsenal. Nat Rev Immunol. 2005;5(4):331-342.

7. Nakahira K, et al. Autophagy proteins regulate innate immune responses by inhibiting the release of mitochondrial DNA mediated by the NALP3 inflammasome. Nat Immunol. 2011;12(3):222-230.

8. Nakahira K, et al. Circulating mitochondrial DNA in patients in the ICU as a marker of mortality: derivation and validation. PLoS Med. 2013;10(12):e1001577.

9. Ma KC, Schenck EJ, Pabon MA, Choi AMK. The role of danger signals in the pathogenesis and perpetuation of critical illness. Am J Respir Crit Care Med. 2018;197(3):300-309. 
10. Hotchkiss RS, et al. Apoptotic cell death in patients with sepsis, shock, and multiple organ dysfunction. Crit Care Med. 1999;27(7):1230-1251.

11. Hotchkiss RS, Karl IE. The pathophysiology and treatment of sepsis. N Engl J Med. 2003;348(2):138-150

12. Linkermann A, Green DR. Necroptosis. N Engl J Med. 2014;370(5):455-465.

13. Newton K, et al. Activity of protein kinase RIPK3 determines whether cells die by necroptosis or apoptosis. Science. 2014;343(6177):1357-1360.

14. Moreno-Gonzalez G, Vandenabeele P, Krysko DV. Necroptosis: A novel cell death modality and its potential relevance for critical care medicine. Am J Respir Crit Care Med. 2016;194(4):415-428.

15. Qing DY, et al. Red blood cells induce necroptosis of lung endothelial cells and increase susceptibility to lung inflammation. $A m$ J Respir Crit Care Med. 2014;190(11):1243-1254

16. Siempos II, et al. RIPK3 mediates pathogenesis of experimental ventilator-induced lung injury. JCI Insight. 2018;3(9):e97102.

17. Shashaty MG, et al. Plasma levels of receptor interacting protein kinase-3 (RIP3), an essential mediator of necroptosis, are associated with acute kidney injury in critically ill trauma patients. Shock. 2016;46(2):139-143.

18. Ma KC, et al. Increased receptor interacting serine/threonine-protein kinase 3 plasma levels is associated with severity of sepsis American Thoracic Society. https://www.atsjournals.org/doi/abs/10.1164/ajrccm-conference.2016.193.1_MeetingAbstracts. A2724. Accessed June 11, 2018

19. Patino E, Ma KC, Sureshbabu A, Finkelzstein E, Choi AMK, Choi M. Increased plasma and urine RIPK3 levels correlate with acute kidney injury and predict duration of kidney dysfunction during sepsis. American Thoracic Society. https://www.atsjournals.org/doi/abs/10.1164/ajrccm-conference.2017.195.1_MeetingAbstracts.A7139. Accessed June 11, 2018.

20. Hotchkiss RS, et al. Rapid onset of intestinal epithelial and lymphocyte apoptotic cell death in patients with trauma and shock Crit Care Med. 2000;28(9):3207-3217.

21. Vanden Berghe T, Linkermann A, Jouan-Lanhouet S, Walczak H, Vandenabeele P. Regulated necrosis: the expanding network of non-apoptotic cell death pathways. Nat Rev Mol Cell Biol. 2014;15(2):135-147.

22. Linkermann A, Stockwell BR, Krautwald S, Anders HJ. Regulated cell death and inflammation: an auto-amplification loop causes organ failure. Nat Rev Immunol. 2014;14(11):759-767.

23. Wegner KW, Saleh D, Degterev A. Complex pathologic roles of RIPK1 and RIPK3: moving beyond necroptosis. Trends Phar macol Sci. 2017;38(3):202-225.

24. Mandal P, et al. RIP3 induces apoptosis independent of pronecrotic kinase activity. Mol Cell. 2014;56(4):481-495.

25. Cook WD, et al. RIPK1- and RIPK3-induced cell death mode is determined by target availability. Cell Death Differ. 2014;21(10):1600-1612.

26. Lawlor KE, et al. RIPK3 promotes cell death and NLRP3 inflammasome activation in the absence of MLKL. Nat Commun. $2015 ; 6: 6282$.

27. Davenport EE, et al. Genomic landscape of the individual host response and outcomes in sepsis: a prospective cohort study. Lancet Respir Med. 2016;4(4):259-271.

28. Finkelsztein EJ, et al. Comparison of qSOFA and SIRS for predicting adverse outcomes of patients with suspicion of sepsis outside the intensive care unit. Crit Care. 2017;21(1):73.

29. Dolinay T, et al. Inflammasome-regulated cytokines are critical mediators of acute lung injury. Am J Respir Crit Care Med. 2012;185(11):1225-1234.

30. Park J, et al. Plasma surfactant protein-D as a diagnostic biomarker for acute respiratory distress syndrome: validation in US and Korean cohorts. BMC Pulm Med. 2017;17(1):204.

31. Nakahira K, Hisata S, Choi AM. The roles of mitochondrial damage-associated molecular patterns in diseases. Antioxid Redox Signal. 2015;23(17):1329-1350.

32. Knaus WA, Draper EA, Wagner DP, Zimmerman JE. APACHE II: a severity of disease classification system. Crit Care Med. $1985 ; 13(10): 818-829$.

33. Vincent JL, et al. The SOFA (sepsis-related organ failure assessment) score to describe organ dysfunction/failure. On behalf of the Working Group on Sepsis-Related Problems of the European Society of Intensive Care Medicine. Intensive Care Med. 1996;22(7):707-710 